\title{
Theoretical Approach to Strongly Correlated Systems Based on the U(1) and SU(2) Symmetry Groups
}

\author{
T.K. KOPEĆ \\ Institute for Low Temperature and Structure Research, Polish Academy of Sciences \\ POB 1410, 50-950 Wroclaw 2, Poland
}

\begin{abstract}
The spin-rotationally invariant $\mathrm{SU}(2) \times \mathrm{U}(1)$ approach to the Hubbard model is extended to accommodate the charge degrees of freedom. Both $\mathrm{U}(1)$ and $\mathrm{SU}(2)$ gauge transformation are used to factorize the charge and spin contribution to the original electron operator in terms of the emergent gauge fields. By tracing out gauge bosons the form of paired states is established and the role of antiferromagnetic correlations is explicated. We argue that in strongly correlated electron system collective instanton excitations of the phase field (dual to the charge) arise with a great degree of stability, governed by gauge flux changes by an integer multiple of $2 \pi$. Furthermore, it is shown that $\mathrm{U}(1)$ and $\mathrm{SU}(2)$ gauge fields play a similar role as phonons in the BCS theory: they act as the the "glue" for fermion pairing.
\end{abstract}

PACS: 74.20.-z, 74.20.Fg, 71.10.Pm

\section{Introduction}

It is believed that the ultimate complication on the study of electronic properties of the many-body systems is due to the inter-particle interactions. Sometimes, one can deal away this problem by invoking the Fermi-liquid (FL) theory [1], but there is a number of examples including e.g. superfluidity, high temperature superconductivity, and the fractional quantum Hall effect, where quantum coherence manifest in various dramatic ways and there is a very difficult task to describe them with the help of the independent particle picture. A major complication in dealing with interaction effects is the notorious complexity of the underlying theory. Among the electronic Hamiltonians relevant for interacting systems the Hubbard model [2] and $t-J$ model as its descendant [3] are considered as those that contains the essential ingredients for understanding the physics of correlated electrons. An essential pre-requisite to the construction of the theory is a solid understanding of the fundamental symmetries involved in the problem under study. In a many-body system boundary conditions are encoded in the symmetries and in the Hubbard model they are represented by the charge $\mathrm{U}(1)$ gauge and spin rotational $\mathrm{SU}(2)$ groups relevant for the occurrence of the superconducting and magnetic orderings. The symmetry related boundary conditions superimposed on the manybody wavefunction are than reflected in the topological structure of the configuration space. In particular for multiply connected configuration spaces novel features can arise as documented for example by the AharonovBöhm effect [4] governed by the multiply connected U(1) group manifold.
Following Feynman path integral description of the quantum mechanics [5] new possibilities arise when the space of trajectories falls into disconnected pieces and the essential question is how to weight the different path. When the homotopy class of the symmetry group governing the quantum dynamics in non-trivial as the for the multiply connected $\mathrm{U}(2)=\mathrm{U}(1) \otimes \mathrm{SU}(2)$ group manifold pertinent for the Hubbard model, the amplitude assigned to a trajectory depends not only on permutations experienced by particles as the follow the trajectory but also on other aspect of their paths by which they wind around one another. Since the homotopy class $\pi_{1}[\mathrm{U}(2)]=Z$ forms a set of integer winding numbers the topological structure of the configuration space is nontrivial, ambiguities may arise when attempts are made to specify a value for the phase of a wavefunction for the whole configuration space. Thus the problem we are facing is that of many-body quantum mechanics on a multiply connected configuration space. According to the general rules of Feynman path integrals in multiply connected configuration space, one has divide the space of paths into homotopy classes parametrized by winding numbers, and rewrite the path integral as a sum of subintegrals, for each of which such class, respectively [6].

In the present paper we putt this program into practice and develop a spin-charge unifying description for interacting electrons given by the Hubbard model. It is based on the time dependent local gauge transformations to disentangle the Coulomb interaction. The collective variables for charge and spin are isolated in a form of the space-time fluctuating $\mathrm{U}(1)$ phase field and the rotating spin quantization axis governed by the $\mathrm{SU}(2)$ symmetry, respectively. As a result interacting electrons appear as 
composite objects consisting of bare fermions with attached U(1) and $\mathrm{SU}(2)$ gauge fields. Finally, we unravel the link between the non-trivial topological structure of the resulting $\mathrm{U}(2)=\mathrm{U}(1) \otimes \mathrm{SU}(2)$ configurational space for gauge fields and the novel type of quantum criticality.

\section{Hubbard Hamiltonian}

Our starting point is the purely fermionic Hubbard Hamiltonian $\mathcal{H} \equiv \hat{\mathcal{H}}_{t}+\hat{\mathcal{H}}_{U}$ :

$$
\begin{aligned}
\hat{\mathcal{H}} & =-t \sum_{\left\langle\boldsymbol{r} \boldsymbol{r}^{\prime}\right\rangle, \alpha}\left(\hat{c}_{\alpha}^{\dagger}(\boldsymbol{r}) \hat{c}_{\alpha}\left(\boldsymbol{r}^{\prime}\right)+\text { h.c. }\right) \\
& +U \sum_{\boldsymbol{r}} \hat{n}_{\uparrow}(\boldsymbol{r}) \hat{n}_{\downarrow}(\boldsymbol{r}) .
\end{aligned}
$$

Here, $\left\langle\boldsymbol{r}, \boldsymbol{r}^{\prime}\right\rangle$ runs over the nearest-neighbor (n.n.) sites, $t$ is the hopping amplitude, $U$ stands for the Coulomb repulsion, while the operator $\hat{c}_{\alpha}^{\dagger}(\boldsymbol{r})$ creates an electron with spin $\alpha=\uparrow, \downarrow$ at the lattice site $\boldsymbol{r}$, where $\hat{n}_{\alpha}(\boldsymbol{r})=$ $\hat{c}_{\alpha}^{\dagger}(\boldsymbol{r}) \hat{c}_{\alpha}(\boldsymbol{r})$. Usually, working in the grand canonical ensemble a term is added to $\hat{\mathcal{H}}$ in Eq. (1) to control the average number of electrons,

$$
\hat{\mathcal{H}} \rightarrow \hat{\mathcal{H}}-\mu \sum_{\boldsymbol{r}} \hat{n}(\boldsymbol{r}),
$$

with $\mu$ being the chemical potential and $\hat{n}(\boldsymbol{r})=\hat{n}_{\uparrow}(\boldsymbol{r})+$ $\hat{n}_{\downarrow}(\boldsymbol{r})$ the number operator. It is customary to introduce Grassmann fields $c_{\alpha}(\boldsymbol{r} \tau)$ depending on the "imaginary time" $0 \leq \tau \leq \beta \equiv 1 / k_{B} T$, (with $T$ being the temperature) that satisfy the anti-periodic condition $c_{\alpha}(\boldsymbol{r} \tau)=-c_{\alpha}(\boldsymbol{r} \tau+\beta)$, to write the path integral for the statistical sum $\mathcal{Z}=\int[\mathcal{D} \bar{c} \mathcal{D} c] \mathrm{e}^{-\mathcal{S}[\bar{c}, c]}$ with the fermionic action

$$
\mathcal{S}[\bar{c}, c]=\mathcal{S}_{B}[\bar{c}, c]+\int_{0}^{\beta} \mathrm{d} \tau \mathcal{H}[\bar{c}, c],
$$

that contains the fermionic Berry term

$$
\mathcal{S}_{B}[\bar{c}, c]=\sum_{\boldsymbol{r}_{\alpha}} \int_{0}^{\beta} \mathrm{d} \tau \bar{c}_{\alpha}(\boldsymbol{r} \tau) \partial_{\tau} c_{\alpha}(\boldsymbol{r} \tau) .
$$

that will paly an important role in our considerations.

\section{Spin-charge reference frames}

The standard scheme for dealing with interacting electrons is to employ the Hubbard-Stratonovich transformation followed by a saddle-point analysis. It turns out that a straightforward implementation of this approach is beset with a number of problems. However, these difficulties can be circumvent will in a scheme that is firmly rooted in the gauge symmetries of the Hubbard model.

\subsection{Rotating $S U(2)$ spin reference frame}

In order to maintain spin rotational invariance, one should consider the spin-quantization axis to be a priori arbitrary and integrate over all possible directions in the partition function. For this purpose the density-density product in Eq. (1) we write, following Ref. [7], in a spinrotational invariant way:

$$
\mathcal{H}_{U}=U \sum_{\boldsymbol{r}}\left(\frac{1}{4} n^{2}(\boldsymbol{r} \tau)-(\boldsymbol{\Omega}(\boldsymbol{r} \tau) \cdot \boldsymbol{S}(\boldsymbol{r} \tau))^{2}\right),
$$

where $S^{a}(\boldsymbol{r} \tau)=\frac{1}{2} \sum_{\alpha \alpha^{\prime}} c_{\alpha}^{\dagger}(\boldsymbol{r} \tau) \sigma_{\alpha \alpha^{\prime}}^{a} c_{\alpha^{\prime}}(\boldsymbol{r} \tau)$ denotes the vector spin operator $(a=x, y, z)$ with $\sigma^{a}$ being the Pauli matrices. The unit vector

$$
\begin{aligned}
& \boldsymbol{\Omega}(\boldsymbol{r} \tau)=[\sin \vartheta(\boldsymbol{r} \tau) \cos \varphi(\boldsymbol{r} \tau), \sin \vartheta(\boldsymbol{r} \tau) \sin \varphi(\boldsymbol{r} \tau), \\
& \quad \cos \vartheta(\boldsymbol{r} \tau)],
\end{aligned}
$$

written in terms of polar angles labels varying in spacetime spin quantization axis. The spin-rotation invariance is made explicit by performing the angular integration over $\boldsymbol{\Omega}(\boldsymbol{r} \tau)$ at each site and time. By decoupling spin and charge density terms in Eq. (5) using auxiliary fields $\varrho(\boldsymbol{r} \tau)$ and i $V(\boldsymbol{r} \tau)$ respectively, we write down the partition function in the form

$$
\mathcal{Z}=\int[\mathcal{D} \boldsymbol{\Omega}] \int[\mathcal{D} V \mathcal{D} \varrho] \int[\mathcal{D} \bar{c} \mathcal{D} c] \mathrm{e}^{-\mathcal{S}[\boldsymbol{\Omega}, V, \varrho, \bar{c}, c]},
$$

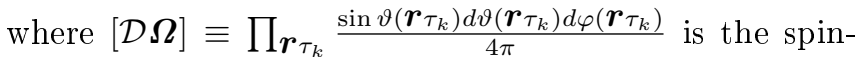
angular integration measure. The effective action reads:

$$
\begin{aligned}
\mathcal{S} & {[\boldsymbol{\Omega}, V, \varrho, \bar{c}, c]=\sum_{\boldsymbol{r}} \int_{0}^{\beta} \mathrm{d} \tau\left(\frac{\varrho^{2}(\boldsymbol{r} \tau)}{U}+\frac{V^{2}(\boldsymbol{r} \tau)}{U}\right.} \\
& +\mathrm{i} V(\boldsymbol{r} \tau) n(\boldsymbol{r} \tau)+2 \varrho(\boldsymbol{r} \tau) \boldsymbol{\Omega}(\boldsymbol{r} \tau) \cdot \boldsymbol{S}(\boldsymbol{r} \tau)) \\
& +\mathcal{S}_{B}[\bar{c}, c]+\int_{0}^{\beta} \mathrm{d} \tau \mathcal{H}_{t}[\bar{c}, c] .
\end{aligned}
$$

\section{2. $U(1)$ rotor charge frame}

To isolate strongly fluctuating modes generated by the Hubbard term according to the charge U(1) symmetry we write the fluctuating "imaginary chemical potential" i $V(\boldsymbol{r} \tau)$ as a sum of a static $V_{0}(\boldsymbol{r})$ and periodic function $V(\boldsymbol{r} \tau)=V_{0}(\boldsymbol{r})+\tilde{V}(\boldsymbol{r} \tau)$ using Fourier series

$$
\tilde{V}(\boldsymbol{r} \tau)=\frac{1}{\beta} \sum_{n=1}^{\infty}\left(\tilde{V}\left(\boldsymbol{r} \omega_{n}\right) \mathrm{e}^{\mathrm{i} \omega_{n} \tau}+\text { c.c. }\right),
$$

with $\omega_{n}=2 \pi n / \beta(n=0, \pm 1, \pm 2)$ being the (Bose) Matsubara frequencies. Now, we introduce the $\mathrm{U}(1)$ phase field $\phi(\boldsymbol{r} \tau)$ via the Faraday-type relation

$$
\dot{\phi}(\boldsymbol{r} \tau) \equiv \frac{\partial \phi(\boldsymbol{r} \tau)}{\partial \tau}=\mathrm{e}^{-\mathrm{i} \phi(\boldsymbol{r} \tau)} \frac{1}{\mathrm{i}} \frac{\partial}{\partial \tau} \mathrm{e}^{\mathrm{i} \phi(\boldsymbol{r} \tau)}=\tilde{V}(\boldsymbol{r} \tau) .
$$

Furthermore, by performing the local gauge transformation to the new fermionic variables $f_{\alpha}(\boldsymbol{r} \tau)$ :

$$
\left[\begin{array}{c}
c_{\alpha}(\boldsymbol{r} \tau) \\
\bar{c}_{\alpha}(\boldsymbol{r} \tau)
\end{array}\right]=\left[\begin{array}{cc}
z(\boldsymbol{r} \tau) & 0 \\
0 & \bar{z}(\boldsymbol{r} \tau)
\end{array}\right]\left[\begin{array}{c}
f_{\alpha}(\boldsymbol{r} \tau) \\
\bar{f}_{\alpha}(\boldsymbol{r} \tau)
\end{array}\right],
$$

where the unimodular parameter $|z(\boldsymbol{r} \tau)|^{2}=1$ satisfies $z(\boldsymbol{r} \tau)=\mathrm{e}^{\mathrm{i} \phi(\boldsymbol{r} \tau)}$, we remove the imaginary term i $\int_{0}^{\beta} \mathrm{d} \tau \tilde{V}(\boldsymbol{r} \tau) n(\boldsymbol{r} \tau)$ for all the Fourier modes of the $V(\boldsymbol{r} \tau)$ field, except for the zero frequency. Subsequent $\mathrm{SU}(2)$ transformation from $f_{\alpha}(\boldsymbol{r} \tau)$ to $h_{\alpha}(\boldsymbol{r} \tau)$ operators,

$$
\left[\begin{array}{l}
f_{1}(\boldsymbol{r} \tau) \\
f_{2}(\boldsymbol{r} \tau)
\end{array}\right]=\left[\begin{array}{cc}
\zeta_{1}(\boldsymbol{r} \tau) & -\bar{\zeta}_{2}(\boldsymbol{r} \tau) \\
\zeta_{2}(\boldsymbol{r} \tau) & \bar{\zeta}_{1}(\boldsymbol{r} \tau)
\end{array}\right]\left[\begin{array}{c}
h_{1}(\boldsymbol{r} \tau) \\
h_{2}(\boldsymbol{r} \tau)
\end{array}\right],
$$

with the constraint $\left|\zeta_{1}(\boldsymbol{r} \tau)\right|^{2}+\left|\zeta_{2}(\boldsymbol{r} \tau)\right|^{2}=1$ takes away the rotational dependence on $\boldsymbol{\Omega}(\boldsymbol{r} \tau)$ in the spin sector. 
This is doneby means of the Hopf map

$$
\boldsymbol{R}(\boldsymbol{r} \tau) \sigma^{z} \boldsymbol{R}^{\dagger}(\boldsymbol{r} \tau)=\sigma \cdot \boldsymbol{\Omega}(\boldsymbol{r} \tau),
$$

that is based on the enlargement from two-sphere $S_{2}$ to the three-sphere $S_{3} \sim S U(2)$. The unimodular constraint can be resolved by using the parametrization

$$
\begin{aligned}
& \zeta_{1}(\boldsymbol{r} \tau)=\mathrm{e}^{-\frac{\mathrm{i}}{2}\left(\varphi\left(\boldsymbol{r}_{\tau}\right)+\chi\left(\boldsymbol{r}_{\tau}\right)\right)} \cos \left(\frac{\vartheta(\boldsymbol{r} \tau)}{2}\right), \\
& \zeta_{2}(\boldsymbol{r} \tau)=\mathrm{e}^{\frac{\mathrm{i}}{2}\left(\varphi\left(\boldsymbol{r}_{\tau}\right)-\chi\left(\boldsymbol{r}_{\tau}\right)\right)} \sin \left(\frac{\vartheta(\boldsymbol{r} \tau)}{2}\right),
\end{aligned}
$$

with the Euler angular variables $\varphi(\boldsymbol{r} \tau), \vartheta(\boldsymbol{r} \tau)$ and $\chi(\boldsymbol{r} \tau)$, respectively. Here, the extra variable $\chi(\boldsymbol{r} \tau)$ represents the $\mathrm{U}(1)$ gauge freedom of the theory as a consequence of $S_{2} \rightarrow S_{3}$ mapping. One can summarize Eqs. (11) and (12) by the single joint gauge transformation exhibiting electron operator factorization

$$
c_{\alpha}(\boldsymbol{r} \tau)=\sum_{\alpha^{\prime}} \mathcal{U}_{\alpha \alpha^{\prime}}(\boldsymbol{r} \tau) h_{\alpha^{\prime}}(\boldsymbol{r} \tau),
$$

where

$$
\mathcal{U}(\boldsymbol{r} \tau)=z(\boldsymbol{r} \tau) \boldsymbol{R}(\boldsymbol{r} \tau)
$$

is a $\mathrm{U}(2)$ matrix matrix which rotates the spinquantization axis at site $\boldsymbol{r}$ and time $\tau$. Eq. (15) reflects the composite nature of the interacting electron formed from bosonic spinorial and charge degrees of freedom given by $R_{\alpha \alpha^{\prime}}(\boldsymbol{r} \tau)$ and $z(\boldsymbol{r} \tau)$, respectively as well as remaining fermionic part $h_{\alpha}(\boldsymbol{r} \tau)$.

\section{Effective phase-angular action}

In this section, we define a path integral representation of the partition function. The introduction of a fluctuating spin-quantization axis and phase field for the charge in the functional integral allows us to consider spin and charge fluctuations on equal footing.

\subsection{Tracing massive variables}

The expectation value of the static (zero frequency) part of the fluctuating electrochemical potential $V_{0}(\boldsymbol{r})$ we calculate by the saddle point method to give

$$
V_{0}(\boldsymbol{r})=\mathrm{i}\left(\mu-\frac{U}{2} n_{h}\right) \equiv \mathrm{i} \bar{\mu},
$$

where $\bar{\mu}$ is the chemical potential with a Hartree shift originating from the saddle-point value of the static variable $V_{0}(\boldsymbol{r})$ with $n_{h}=n_{h \uparrow}+n_{h \downarrow}$ and $n_{h \alpha}=$ $\left\langle\bar{h}_{\alpha}(\boldsymbol{r} \tau) h_{\alpha}(\boldsymbol{r} \tau)\right\rangle$. Similarly in the magnetic sector

$$
\rho(\boldsymbol{r} \tau)=\left\{\begin{array}{r}
(-1)^{\boldsymbol{r}} \Delta_{c} \\
\pm \Delta_{c}
\end{array},\right.
$$

where $\Delta_{c}=U\left\langle S^{z}(\boldsymbol{r} \tau)\right\rangle$ sets the magnitude for the Mottcharge gap [8]. The two choices delineated in Eq. (18) correspond to the saddle point of the "antifferomagnetic" (with staggering $\Delta_{c}$ ) or "ferromagnetic type". Note that the notion ferromagnetic (antifferomagnetic)here does not mean an actual long-range ordering - for this the angular spin-quantization variables have to be ordered as well. In the new variables the action in Eq. (8) assumes the form

$$
\begin{aligned}
\mathcal{S} & {[\boldsymbol{\Omega}, \phi, \varrho, \bar{h}, h]=\mathcal{S}_{B}[\bar{h}, h]+\int_{0}^{\beta} \mathrm{d} \tau \mathcal{H}_{\boldsymbol{\Omega}, \phi}[\rho, \bar{h}, h] } \\
& +\mathcal{S}_{0}[\phi]+2 \sum_{\boldsymbol{r}} \int_{0}^{\beta} \mathrm{d} \tau \boldsymbol{A}(\boldsymbol{r} \tau) \cdot \boldsymbol{S}_{h}(\boldsymbol{r} \tau),
\end{aligned}
$$

where $\boldsymbol{S}_{h}(\boldsymbol{r} \tau)=\frac{1}{2} \sum_{\alpha \gamma} \bar{h}_{\alpha}(\boldsymbol{r} \tau) \sigma_{\alpha \gamma} h_{\gamma}(\boldsymbol{r} \tau)$. Furthermore,

$$
S_{0}[\phi]=\sum_{\boldsymbol{r}} \int_{0}^{\beta} \mathrm{d} \tau\left(\frac{\dot{\phi}^{2}(\boldsymbol{r} \tau)}{U}+\frac{1}{\mathrm{i}} \frac{2 \mu}{U} \dot{\phi}(\boldsymbol{r} \tau)\right),
$$

stands for the kinetic and Berry term of the U(1) phase field in the charge sector. The $\mathrm{SU}(2)$ gauge transformation in Eq. (12) and the fermionic Berry term in Eq. (4) generate $\mathrm{SU}(2)$ potentials given by

$$
\begin{aligned}
& \boldsymbol{R}^{\dagger}(\boldsymbol{r} \tau) \partial_{\tau} \boldsymbol{R}(\boldsymbol{r} \tau)=\boldsymbol{R}^{\dagger}\left(\dot{\varphi} \frac{\partial}{\partial \varphi}+\dot{\vartheta} \frac{\partial}{\partial \vartheta}+\dot{\chi} \frac{\partial}{\partial \chi}\right) \boldsymbol{R} \\
& \quad=-\sigma \cdot \boldsymbol{A}(\boldsymbol{r} \tau),
\end{aligned}
$$

where

$$
\begin{aligned}
& A^{x}(\boldsymbol{r} \tau)=\frac{\mathrm{i}}{2} \dot{\vartheta}(\boldsymbol{r} \tau) \sin \chi(\boldsymbol{r} \tau) \\
& -\frac{\mathrm{i}}{2} \dot{\varphi}(\boldsymbol{r} \tau) \sin \theta(\boldsymbol{r} \tau) \cos \chi(\boldsymbol{r} \tau), \\
& A^{y}(\boldsymbol{r} \tau)=\frac{\mathrm{i}}{2} \dot{\vartheta}(\boldsymbol{r} \tau) \cos \chi(\boldsymbol{r} \tau) \\
& \quad+\frac{\mathrm{i}}{2} \dot{\varphi}(\boldsymbol{r} \tau) \sin \theta(\boldsymbol{r} \tau) \sin \chi(\boldsymbol{r} \tau) \\
& A^{z}(\boldsymbol{r} \tau)=\frac{\mathrm{i}}{2} \dot{\varphi}(\boldsymbol{r} \tau) \cos \vartheta(\boldsymbol{r} \tau)+\frac{\mathrm{i}}{2} \dot{\chi}(\boldsymbol{r} \tau),
\end{aligned}
$$

are the $\mathrm{SU}(2)$ gauge potentials.

\subsection{Fermionic action}

The fermionic sector, in turn, is governed by the effective Hamiltonian

$$
\begin{aligned}
& \mathcal{H}_{\boldsymbol{\Omega}, \phi}=\sum_{\boldsymbol{r}} \varrho(\boldsymbol{r} \tau)\left[\bar{h}_{\uparrow}(\boldsymbol{r} \tau) h_{\uparrow}(\boldsymbol{r} \tau)-\bar{h}_{\downarrow}(\boldsymbol{r} \tau) h_{\downarrow}(\boldsymbol{r} \tau)\right] \\
&-t \sum_{\left\langle\boldsymbol{r}, \boldsymbol{r}^{\prime}\right\rangle} \sum_{\alpha \gamma}\left[\mathcal{U}^{\dagger}(\boldsymbol{r} \tau) \mathcal{U}\left(\boldsymbol{r}^{\prime} \tau\right)\right]_{\alpha \gamma} \bar{h}_{\alpha}(\boldsymbol{r} \tau) h_{\gamma}\left(\boldsymbol{r}^{\prime} \tau\right) \\
&-\bar{\mu} \sum_{\boldsymbol{r}_{\alpha}} \bar{h}_{\alpha}(\boldsymbol{r} \tau) h_{\alpha}(\boldsymbol{r} \tau),
\end{aligned}
$$

The result of the gauge transformations is that we have managed to cast the strongly correlated problem into a system of mutually non-interacting fermions, submerged in the bath of strongly fluctuating $\mathrm{U}(1)$ and $\mathrm{SU}(2)$ fields whose dynamics is governed by the energy scale set by the Coulomb interaction $U$ coupled to fermions via hopping term and with the Zeeman-type contribution with the massive field $\varrho(\boldsymbol{r} \tau)$.

In analogy to the charge $\mathrm{U}(1)$ field the $\mathrm{SU}(2)$ spin system exhibit emergent dynamics. By integrating out fermions the last term in Eq. (19) will generate the kinetic term for the $\mathrm{SU}(2)$ rotors 


$$
\begin{aligned}
& \mathcal{S}_{0}[\boldsymbol{\Omega}]=-\frac{1}{2} 4 \sum_{\boldsymbol{r} \boldsymbol{r}^{\prime}} \int_{0}^{\beta} \mathrm{d} \tau d \tau^{\prime} \sum_{a b}\left\langle A^{a}(\boldsymbol{r} \tau) A^{b}\left(\boldsymbol{r}^{\prime} \tau^{\prime}\right)\right\rangle \\
& \quad \times \sum_{\boldsymbol{r}^{\prime}}\left\langle S_{h}^{a}(\boldsymbol{r} \tau) S_{h}^{b}\left(\boldsymbol{r}^{\prime} \tau^{\prime}\right)\right\rangle \\
& \quad-2 \sum_{\boldsymbol{r} \boldsymbol{r}^{\prime}} \int_{0}^{\beta} \mathrm{d} \tau\left\langle\boldsymbol{A}(\boldsymbol{r} \tau) \cdot \boldsymbol{S}_{h}\left(\boldsymbol{r}^{\prime} \tau^{\prime}\right)\right\rangle,
\end{aligned}
$$

with

$$
\begin{aligned}
& \left\langle S_{h}^{a}(\boldsymbol{r} \tau) S_{h}^{b}\left(\boldsymbol{r}^{\prime} \tau^{\prime}\right)\right\rangle=-\frac{1}{4} 2 \delta_{a b} \frac{1}{\mathcal{E}_{s}}, \\
& \left\langle S_{h}^{z}(\boldsymbol{r} \tau)\right\rangle=\operatorname{Tr}\left(\sigma^{z} G(\boldsymbol{r} \tau, \boldsymbol{r} \tau)\right)=\frac{\Delta_{c}}{U} .
\end{aligned}
$$

For example, in the antifferomagnetic (AF) phase, at the half-filling, it assumes the staggered form $\varrho(\boldsymbol{r} \tau)=$ $\Delta_{c}(-1)^{\boldsymbol{r}}$ with $\Delta_{c}$ being the charge gap $\Delta_{c} \approx U / 2$ for $U / t \gg 1$. At zero temperature

$$
\lim _{T \rightarrow 0} \frac{1}{\mathcal{E}_{s}}=\frac{\Delta_{c}^{2}}{2 E_{\boldsymbol{k}}^{3}},
$$

so that, in the limit $U / t \gg 1$ one obtains $\mathcal{E}_{s} \approx 2 \Delta_{c}=U$. However, a nonzero value of $\Delta_{c}$ does not imply the existence of AF long-range order. For this the angular degrees of freedom $\boldsymbol{\Omega}(\boldsymbol{r} \tau)$ have also to be ordered, whose low-lying excitations are in the form of spin waves. Therefore the kinetic term in the spin sector becomes

$$
\mathcal{S}_{0}[\boldsymbol{\Omega}]=-\frac{1}{\mathcal{E}_{s}} \sum_{\boldsymbol{r}} \int_{0}^{\beta} \mathrm{d} \tau\langle\boldsymbol{A}(\boldsymbol{r} \tau) \cdot \boldsymbol{A}(\boldsymbol{r} \tau)\rangle,
$$

so that the total kinetic energy $\mathcal{S}[\boldsymbol{\Omega}, \phi]=\mathcal{S}_{0}[\phi]+\mathcal{S}_{0}[\boldsymbol{\Omega}]$ for $\mathrm{U}(1)$ and $\mathrm{SU}(2)$ rotors is

$$
\begin{aligned}
\mathcal{S}_{0} & {[\boldsymbol{\Omega}, \phi]=\sum_{\boldsymbol{r}} \int_{0}^{\beta} \mathrm{d} \tau\left(\frac{\dot{\phi}^{2}(\boldsymbol{r} \tau)}{U}+\frac{1}{\mathrm{i}} \frac{2 \mu}{U} \dot{\phi}(\boldsymbol{r} \tau)\right.} \\
& +\frac{1}{4 \mathcal{E}_{s}}\left(\dot{\vartheta}^{2}(\boldsymbol{r} \tau)+\dot{\varphi}^{2}(\boldsymbol{r} \tau)+\dot{\chi}^{2}(\boldsymbol{r} \tau)\right. \\
& +2 \dot{\varphi}(\boldsymbol{r} \tau) \dot{\chi}(\boldsymbol{r} \tau) \cos \vartheta(\boldsymbol{r} \tau)) \\
& \left.+\frac{\Delta_{c}}{\mathrm{i} U}(\dot{\varphi}(\boldsymbol{r} \tau) \cos \vartheta(\boldsymbol{r} \tau)+\dot{\chi}(\boldsymbol{r} \tau))\right) .
\end{aligned}
$$

The distinctive feature of Eq.(28) is th presence of the geometric Berry contributions that signify topological features of the underlying field theory.

\section{Topological features}

\subsection{Statistical theta terms}

In the preceding paragraphs we have shown that a theory of strongly interacting electrons can be transformed to an equivalent description of weakly interacting fermions which are coupled to the "fluxes" of the strongly fluctuating $\mathrm{U}(2)$ gauge field. In regard to the nonperturbative effects, we realized the presence of an additional parameter, the topological angle $\theta_{c} / 2 \pi \equiv 2 \mu / U$, which related to the chemical potential in a geometric Berry phase term

$$
\mathcal{S}_{B}^{c}=\frac{\theta_{c}}{2 \pi \mathrm{i}} \sum_{\boldsymbol{r}} \int_{0}^{\beta} \mathrm{d} \tau \dot{\phi}(\boldsymbol{r} \tau) .
$$

Since topologically the $\mathrm{U}(2)$ group is equivalent to a circle, the configuration space for the field phase filed consists of topological sectors, each characterized by integer $m$ which is the number of times the phase field $\phi(\boldsymbol{r} \tau)$ winds one goes around the circle boundary. The associated topological effects arise as stable, non-perturbative, collective excitations of the phase field (dual to the charge), which carry novel topological characteristics. These are the winding numbers of $\mathrm{U}(1)$ group: $m(\boldsymbol{r}) \equiv$ $\frac{1}{2 \pi} \int_{0}^{\beta} \mathrm{d} \tau \dot{\phi}(\boldsymbol{r} \tau)$ that become topological conserved quantities. Similarly in the spin sector a Berry phase term arises

$$
\mathcal{S}_{B}^{s}=\frac{\theta_{s}}{2 \pi \mathrm{i}} \sum_{\boldsymbol{r}} \int_{0}^{\beta} \mathrm{d} \tau(\dot{\varphi}(\boldsymbol{r} \tau) \cos \vartheta(\boldsymbol{r} \tau)+\dot{\chi}(\boldsymbol{r} \tau)),
$$

with the theta term $\theta_{s} / 2 \pi=\Delta_{c} / U$ that is related to the Mott gap. Here, the integral of the first term in Eq. (30) has a simple geometrical interpretation as it is equal to a solid angle swept by a unit vector $\boldsymbol{\Omega}(\vartheta, \varphi)$ during its motion.

\subsection{Topological charge and the electron density}

In addition to the Coulomb energy $U$ and temperature, the chemical potential $\mu$ plays a crucial role in Mott transition, since it controls the electron filling $n_{e}$. An immediate implication of the composite nature of the electrons is that the electron occupation number (i.e. the average number of of electrons per site in the $\mathrm{Cu}-\mathrm{O}$ plane)

$$
n_{e}=\frac{1}{N} \sum_{\boldsymbol{r}_{\alpha}}\left\langle\bar{c}_{\alpha}(\boldsymbol{r} \tau) c_{\alpha}(\boldsymbol{r} \tau)\right\rangle
$$

consists of the fermion occupation coming from the fermionic part of the composite and a topological contribution resulting from the "flux-tube" attachment:

$$
\begin{aligned}
& \left\langle\sum_{\alpha} \bar{c}_{\alpha}(\boldsymbol{r} \tau) c_{\alpha}(\boldsymbol{r} \tau)\right\rangle=\left\langle\sum_{\alpha} \bar{f}_{\alpha}(\boldsymbol{r} \tau) f_{\alpha}(\boldsymbol{r} \tau)\right\rangle \\
& \quad+\frac{2}{\mathrm{i} U}\left\langle\frac{\partial \phi(\boldsymbol{r} \tau)}{\partial \tau}\right\rangle .
\end{aligned}
$$

The appearance of the topological contribution in Eq. (32) is not surprising given the fact that "statistical angle" depends on the chemical potential and the occupation number is just its conjugate quantity. Owing that the $\mathrm{U}(1)$ topological charge (the winding number) is given by

$$
\begin{aligned}
& m(\boldsymbol{r})=\frac{1}{2 \pi} \int_{0}^{\beta} \mathrm{d} \tau \dot{\phi}(\boldsymbol{r} \tau) \\
& =\frac{1}{2 \pi} \int_{\phi_{0}(\boldsymbol{r})}^{\phi_{0}(\boldsymbol{r})+2 \pi m(\boldsymbol{r})} \mathrm{d} \phi(\boldsymbol{r} \tau),
\end{aligned}
$$

the mean value of the density of topological charge can be written after performing Legendre transformation as

$$
n_{b}=\frac{2 \mu}{U}+\frac{2}{U}\left\langle\frac{1}{\mathrm{i}} \frac{\partial \phi(\boldsymbol{r} \tau)}{\partial \tau}\right\rangle .
$$

Therefore, the average electron occupation number $n_{e}$ is given by 


$$
n_{e}=n_{f}+n_{b}-\frac{2 \mu}{U} .
$$

In the limit of strong (weak) correlations $n_{e}$ interpolates between topological $n_{b}$ (fermionic $n_{f}$ ) occupation numbers. Clearly, in the large- $U$ limit $\mu \rightarrow n_{f} U / 2$, so that $n_{e} \rightarrow n_{b}$ and the system behaves as governed entirely by density of topological charge.

\section{Pairing interaction}

Now we show that $\mathrm{U}(1)$ and $\mathrm{SU}(2)$ emergent gauge fields, the collective high energy modes in the Hubbard system, take over the task which was carried out by phonons in BCS superconductors. In order to obtain an effective interaction among fermions we have to integrate out all the bosonic modes given by $\bar{z}(\boldsymbol{r} \tau), z\left(\boldsymbol{r}^{\prime} \tau\right)$ and $\boldsymbol{R}^{\dagger}(\boldsymbol{r} \tau), \boldsymbol{R}\left(\boldsymbol{r}^{\prime} \tau\right)$ fields. To this end we write the partition function as $\mathcal{Z}=\int[\mathcal{D} \bar{h} \mathcal{D} h] \mathrm{e}^{-\mathcal{S}[\bar{h}, h]}$, where the effective fermionic action is

$$
S_{\text {eff }}[\bar{h}, h]=-\ln \int[\mathcal{D} \phi \mathcal{D} \boldsymbol{\Omega}] \mathrm{e}^{-S[\boldsymbol{\Omega}, \phi, \bar{h}, h]} .
$$

The expression in Eq. (36) generates a cumulant series when expanded with respect to the hopping variable $t$. As a result of averaging the effective fermionic action can be written as follows:

$$
S_{\text {eff }}[\bar{h}, h]=S_{t}^{(1)}[\bar{h}, h]+S^{(2)}[\bar{h}, h],
$$

where, the hopping term assumes the conventional form that is diagonal in the spin indices

$$
S_{t}^{(1)}[\bar{h}, h]=-\tilde{t} \sum_{\left\langle\boldsymbol{r} \boldsymbol{r}^{\prime}\right\rangle, \alpha} \int_{0}^{\beta} \mathrm{d} \tau \bar{h}_{\alpha}(\boldsymbol{r} \tau) h_{\alpha}\left(\boldsymbol{r}^{\prime} \tau\right),
$$

where $\tilde{t}=t g_{c}(\boldsymbol{d}) g_{s}(\boldsymbol{d})$, is the renormalized hopping, with $g_{c}(\boldsymbol{d})=\left\langle\bar{z}(\boldsymbol{r} \tau) z\left(\boldsymbol{r}^{\prime} \tau\right)\right\rangle$ and $g_{s}(\boldsymbol{d})=\sum_{\alpha}\left\langle\zeta_{\alpha}(\boldsymbol{r} \tau) \zeta_{\alpha}\left(\boldsymbol{r}^{\prime} \tau\right)\right\rangle$ being the Gutzwiller-like charge and spin renormalization factors. The second order term is given by

$$
\begin{gathered}
S^{(2)}[\bar{h}, h]=\sum_{\left\langle\boldsymbol{r} \boldsymbol{r}^{\prime}\right\rangle} \int_{0}^{\beta} \mathrm{d} \tau\left(\gamma_{1} n(\boldsymbol{r} \tau) n\left(\boldsymbol{r}^{\prime} \tau\right)\right. \\
\left.-\gamma_{2} \overline{\mathcal{A}}_{h}\left(\boldsymbol{r} \tau \boldsymbol{r}^{\prime} \tau\right) \mathcal{A}_{h}\left(\boldsymbol{r} \tau \boldsymbol{r}^{\prime} \tau\right)\right),
\end{gathered}
$$

where

$$
\begin{aligned}
& \mathcal{A}_{h}\left(\boldsymbol{r} \tau \boldsymbol{r}^{\prime} \tau\right)=\frac{h_{\uparrow}(\boldsymbol{r} \tau) h_{\downarrow}\left(\boldsymbol{r}^{\prime} \tau\right)-h_{\downarrow}(\boldsymbol{r} \tau) h_{\uparrow}\left(\boldsymbol{r}^{\prime} \tau\right)}{\sqrt{2}}, \\
& \overline{\mathcal{A}}_{h}\left(\boldsymbol{r} \tau \boldsymbol{r}^{\prime} \tau\right)=\frac{\bar{h}_{\downarrow}\left(\boldsymbol{r}^{\prime} \tau\right) \bar{h}_{\uparrow}(\boldsymbol{r} \tau)-\bar{h}_{\uparrow}\left(\boldsymbol{r}^{\prime} \tau\right) \bar{h}_{\downarrow}(\boldsymbol{r} \tau)}{\sqrt{2}},
\end{aligned}
$$

are the bond operators relevant for a singlet pairing.

The rotational invariance of the right-hand side in Eq. (39) is manifest since

$$
\begin{aligned}
& -\overline{\mathcal{A}}_{h}\left(\boldsymbol{r} \tau \boldsymbol{r}^{\prime} \tau\right) \mathcal{A}_{h}\left(\boldsymbol{r} \tau \boldsymbol{r}^{\prime} \tau\right)=\boldsymbol{S}_{h}(\boldsymbol{r} \tau) \cdot \boldsymbol{S}_{h}\left(\boldsymbol{r}^{\prime} \tau\right) \\
& -\frac{1}{4} n_{h}(\boldsymbol{r} \tau) n_{h}\left(\boldsymbol{r}^{\prime} \tau\right) .
\end{aligned}
$$

The coefficients $\gamma_{1}$ and $\gamma_{2}$ are given by

$$
\gamma_{1}=\frac{4 t^{2}}{U}\left(f^{2}(\boldsymbol{d})+\frac{1}{2} g^{2}(\mathbf{0})\right),
$$

$$
\gamma_{2}=\frac{4 t^{2}}{U}\left(3 f^{2}(\boldsymbol{d})\right)
$$

where $f$ and $g$ are the correlation functions:

$$
\begin{aligned}
& g\left(\boldsymbol{r}-\boldsymbol{r}^{\prime}\right)=-\left\langle\zeta_{\alpha}(\boldsymbol{r} \tau) \bar{\zeta}_{\alpha}\left(\boldsymbol{r}^{\prime} \tau\right)\right\rangle, \\
& f\left(\boldsymbol{r}-\boldsymbol{r}^{\prime}\right)=\left\langle\zeta_{\alpha}(\boldsymbol{r} \tau) \zeta_{\alpha}\left(\boldsymbol{r}^{\prime} \tau\right)\right\rangle,
\end{aligned}
$$

that can be readily evaluated using the propagator of the

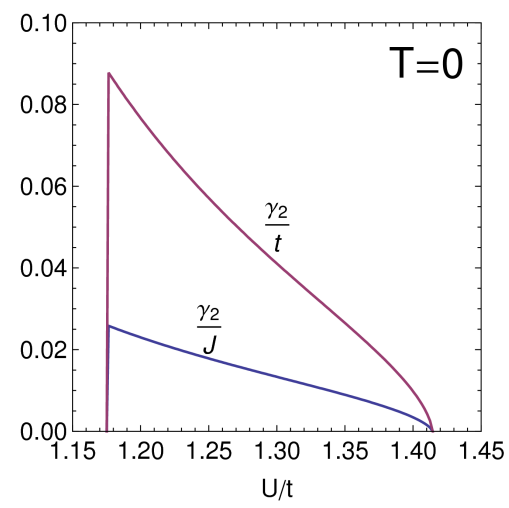

Fig. 1. Pairing interaction $\gamma_{2}$ normalized to the hopping parameter $t$ (upper curve) and the antiferromagnetic exchange parameter $J=4 t^{2} / U$ (lower curve) as a function of $U / t$ calculated at zero temperature and half-filing $\bar{\mu}=0$ for the two-dimensional Hubbard model with nearest-neighbors hopping.

$\zeta$-fields. The effective non-retarded interaction containing $\gamma_{2}$ in front of the $\overline{\mathcal{A}}\left(\boldsymbol{r} \tau \boldsymbol{r}^{\prime} \tau\right) \mathcal{A}\left(\boldsymbol{r} \tau \boldsymbol{r}^{\prime} \tau\right)$ term is negative and therefore constitutes the attractive potential for fermion pairing. We can see that the coefficient $\gamma_{2}$ is not just given by the bare AF exchange $J=4 t^{2} / U$ but is renormalized downwards by the quantity that is related to the antifferomagnetic spin stiffness. The result for $\gamma_{2}$ is plotted in Fig. 1. Note that the pairing interaction survives in rather narrow range of the Coulomb interaction $1.17<U / t<1.41$. This result suggests that superconductivity in the Hubbard model, if possible, represents a rather delicate balance between kinetic energy and Coulomb interaction.

\section{Summary}

For strongly correlated systems the route leading from the microscopic Hamiltonian to the appropriate effective description is rather non trivial. This means that the system has a simple description only in terms of "particles" or other objects, which are very different from the microscopic constituents. In this paper we have observed that the important symmetries of the Hubbard model given by by the charge $\mathrm{U}(1)$ gauge and spin rotational $\mathrm{SU}(2)$ groups, that relevant for the occurrence of the superconducting and magnetic orderings, imply that the quantum dynamics is governed non-trivially by the multiply connected $\mathrm{U}(2)=\mathrm{U}(1) \otimes \mathrm{SU}(2)$ group manifold. As a result interacting electrons appear as a composite objects consisting of bare fermions with attached gauge fields. We 
have obtained the effective action with the Coulomb interaction that contain topological contributions to the effective action. These Berry terms are instrumental for identifying the "quantum protectorates" — stable states of matter whose generic low energy properties are insensitive to microscopics [9]. Therefore, new type of quantum numbers must be invoked to explain topologically induced quantum critical point (QCP) in cuprates and the associated pinning of the chemical potential. Topological effects arise as stable, non-perturbative, collective excitations of the phase field (dual to the charge), which carry novel topological characteristics. These are the winding numbers of U(1) group that become topological conserved quantities. It is exactly the appearance of these topological charges that render the system "protected" against small changes of the hamiltonian's parameters. This novel conservation does not arise just out of a symmetry of the theory (as "conventional" conservation laws based on Noether's theorem) but it is a consequence of the connectedness, i.e. topology of the phase space, related to the topological properties of the associated symmetry group [10]. In conclusion, we presented a field-theoretic description of a microscopic model that reveals an intimate relationship between the spin-SU(2) and charge-U(1) symmetry and pairing. We found that the maximal strength of the effective pairing interaction parameter is observed in a rather narrow range of $U / t$.
Finally we observe that superconductivity demands more than just paired fermions - it also requires phase coherence in the charge sector distinguished by the variables $z(\boldsymbol{r} \tau)=\mathrm{e}^{\mathrm{i} \phi(\boldsymbol{r} \tau)}$ to have the phase stiffnesses that are responsible for the actual superconducting state.

\section{References}

[1] L.D. Landau, Sov. Phys. JETP 3, 920 (1957).

[2] J. Hubbard, Proc. R. Soc. London, Ser. A 276, 238 (1963); M.C. Gutzwiller, Phys. Rev. Lett. 10, 159 (1963); J. Kanamori, Prog. Theor. Phys. 30, 275 (1963).

[3] K.A. Chao, J. Spalek, A.M. Oleś, J. Phys. C: Solid Stete Phys. 10, L271 (1977).

[4] Y. Aharonov, D. Bohm, Phys. Rev. 115, 485 (1959).

[5] V.N. Popov, Functional integrals and collective excitations, Cambridge Univesity Press, 1987.

[6] L.S. Schulman, Techniques and Applications of Path Integration, Wiley, New York 1981.

[7] H.J. Schulz, Phys. Rev. Lett. 65, 2462 (1990).

[8] N.F. Mott, Metal-Insulator Transitions, Taylor\&Francis, London 1990.

[9] T.K. Kopeć, Phys. Rev. B 73, 104505 (2006).

[10] G. Morandi, The Role of Topology in Classical and Quantum Physics, Springer-Verlag, 1992. 